\title{
Light Scattering by a Finite Obstacle and Fano Resonances
}

\author{
Michael I. Tribelsky, ${ }^{1,2, *}$ Sergej Flach, ${ }^{2}$ Andrey E. Miroshnichenko, ${ }^{3,2}$ Andrey V. Gorbach, ${ }^{4}$ and Yuri S. Kivshar ${ }^{3}$ \\ ${ }^{1}$ Moscow State Institute of Radioengineering, Electronics and Automation (Technical University), \\ 78 Vernadskiy Avenue, Moscow 119454, Russia \\ ${ }^{2}$ Max-Planck-Institut für Physik komplexer Systeme, Nöthnitzer Strasse 38, Dresden 01187, Germany \\ ${ }^{3}$ Nonlinear Physics Centre, Research School of Physical Sciences and Engineering, Australian National University, \\ Canberra ACT 0200, Australia \\ ${ }^{4}$ Centre for Photonics and Photonic Materials, Department of Physics, University of Bath, \\ Claverton Down, Bath, BA2 7AY, United Kingdom
}

(Received 1 August 2007; revised manuscript received 30 October 2007; published 30 January 2008)

\begin{abstract}
The conditions for observing Fano resonances at elastic light scattering by a single finite-size obstacle are discussed. General arguments are illustrated by consideration of the scattering by a small (relative to the incident light wavelength) spherical obstacle based upon the exact Mie solution of the diffraction problem. The most attention is paid to recently discovered anomalous scattering. An exactly solvable onedimentional discrete model with nonlocal coupling for simulating diffraction in wave scattering in systems with reduced spatial dimensionality is also introduced and analyzed. Deep connections between the resonances in the continuous and discrete systems are revealed.
\end{abstract}

DOI: 10.1103/PhysRevLett.100.043903

Introduction. - Light scattering by an obstacle is one of the most fundamental problems of electrodynamics; see, e.g., monographs [1,2]. Nowadays the interest in this problem has been increased even more owing to its numerous applications in subwavelength optics, information processing, nanotechnologies, and related fields [3]. Recent studies of resonance scattering by small particles with weak dissipation rates (see, e.g., [4-6] and references therein) have revealed new and unexpected features of this phenomenon, including the inverse hierarchy of optical resonances, complicated near-field structure, unusual frequency and size dependencies, etc., allowing us to name such a scattering anomalous. However, despite the highlighted peculiarities, the physical nature of both normal (Rayleigh) and anomalous scattering is the same: the incident light excites localized electromagnetic modes in a scattering particle [plasmons (polaritons)] oscillating with the frequency of the incident wave $\omega$. The corresponding oscillations of polarization of the particle result in the emission of electromagnetic waves with the same frequency $\omega$ which constitutes the scattered light. Resonances in this picture correspond to the cases when $\omega$ occurs close to (the real part of) the eigenfrequency of one of the localized modes.

The described picture looks analogous to the wellknown phenomenon of Fano resonances in quantum physics [7]. Though the Fano resonances in light scattering are a popular issue, attention has been paid either to the microscopic aspects of the scattering in simple plane geometry [8], or to the scattering by a periodic array of particles (holes in a slab) [9]. In contrast to that, in the present Letter we focus on elastic light scattering by a single finite-size particle. We uncover the basic features of the resonances which are not obscured by cooperative phenomena. It also allows us to inspect the scattering at any values of the
PACS numbers: 42.25.Bs, 42.25.Fx, 76.60.Gv, 78.67.Bf

problem parameters including those corresponding to the anomalous scattering and to clarify the important role of the inverse hierarchy of resonances in the transformation of Lorenzian (Breit-Wigner) resonance lines into typical Fano profiles. The developed approach provides a natural and simple explanation of certain "mysterious" features of the anomalous scattering and related problems, such as, e.g., sharp changes in the scattering diagrams (from preferably forward to backward) upon small changes in $\omega$ in the vicinity of quadrupole resonance [10] and the absence of analogous anomalies at the dipole resonance, a typical asymmetric shape of certain resonance lines obtained in the present Letter (see Fig. 1), and a symmetric, Lorenzian shape of the others, etc.

We also introduce and study an exactly solvable onedimensional (1D) discrete model accounting for all main features of the resonant light scattering by a small particle and exhibiting distinctive Fano resonances. The model simulates wave scattering in an array of coupled waveguides [11], photonic crystals [12], and related systems. A remarkable similarity in the manifestation of the resonances in all these cases helps us to reveal the fundamental links between all such phenomena.

Light scattering by a small spherical particle.-Resonant light scattering by a finite-size particle is accompanied by a two-step transformation: incident plane wave $\rightarrow$ localized resonant electromagnetic modes(s) $\rightarrow$ scattered light. On account of the second step of this transformation (radiative damping) the localized modes have finite lifetimes, being actually quasilocalized even at zero dissipation rate. Note, however, that as long as the scattering of a continuous wave is concerned, losses of energy by the quasilocalized modes are exactly compensated by gain from the incident wave. Then, amplitudes of the modes become time independent. 


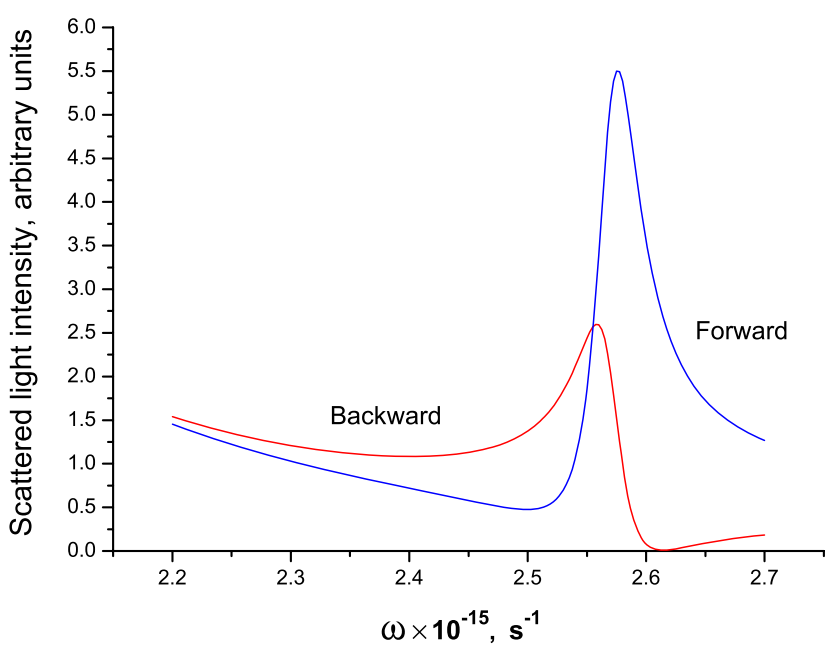

FIG. 1 (color online). An example of typical Fano resonance profiles at elastic light scattering. Forward (blue or dark gray) and backward (red or gray) scattering by a small spherical particle calculated according to the exact Mie solution in the vicinity of the quadrupole resonance; the profiles for $I_{\|}^{(s)}$ and $I_{\perp}^{(s)}$ are identical cf. Eqs. (6) and (7). The media and constants model the sketch of experiment on the anomalous scattering proposed in Ref. [17], namely, a colloidal potassium particle with radius $R=6.2 \times 10^{-6} \mathrm{~cm}$ immersed in a crystal of $\mathrm{KCl}$. The refractive index of the crystal $n_{\mathrm{KCl}}=1.5$; the dielectric permittivity of the particle is approximated by the Drude formula: $\epsilon=$ $1-\omega_{p}^{2} /(\omega+i \nu) \omega$, where $\omega_{p}=5.77 \times 10^{15} \mathrm{~s}^{-1}$ and $\nu=$ $v_{F} / R$ is determined by collisions of free electrons with the particle surface; $v_{F}=10^{8} \mathrm{~cm} / \mathrm{s}$.

Another route for the incident light is just to bypass the scatterer, as well as to excite (nonresonantly) localized modes whose eigenfrequencies lie beyond the close vicinity of $\omega$. Interference of the incident and reemitted light generates a complicated near-field structure and may give rise either to strong enhancement (constructive interference) or strong suppression (destructive interference) of the electromagnetic field. Analogy to the Fano resonances of a quantum particle scattered by a potential with quasidiscrete levels is straightforward. The two possible routes for the incident light correspond to resonant and direct (background) scattering, while the radiative decay of the (quasi)localized modes is identical to tunneling from the quasidiscrete levels.

Though the analogy mentioned is pretty general and valid for any particle size and shape, for the sake of simplicity in what follows we consider light scattering by a small spherical particle described by the exact Mie solution [1,2]. According to the solution for a plane polarized wave propagating along the $z$ axis with vector $\mathbf{E}$ parallel to the $x$ axis the intensities of waves scattered in a given direction are described by the expressions

$$
S_{\|}^{(s)}=I_{\|}^{(s)} \cos ^{2} \varphi ; \quad S_{\perp}^{(s)}=I_{\perp}^{(s)} \sin ^{2} \varphi,
$$

where the subscripts indicate the corresponding polariza- tion (relative to the incident wave). Up to a certain common multiplier quantities $I^{(s)}$ may be expressed as follows:

$$
\begin{aligned}
& I_{\|}^{(s)} \propto\left|\sum_{\ell=1}^{\infty} \frac{2 \ell+1}{\ell(\ell+1)}\left(a_{\ell} P_{\ell}^{(1)^{\prime}}(\cos \theta) \sin \theta-b_{\ell} \frac{P_{\ell}^{(1)}(\cos \theta)}{\sin \theta}\right)\right|^{2}, \\
& I_{\perp}^{(s)} \propto\left|\sum_{\ell=1}^{\infty} \frac{2 \ell+1}{\ell(\ell+1)}\left(a_{\ell} \frac{P_{\ell}^{(1)}(\cos \theta)}{\sin \theta}-b_{\ell} P_{\ell}^{(1)^{\prime}}(\cos \theta) \sin \theta\right)\right|^{2} .
\end{aligned}
$$

Here $\theta$ and $\varphi$ are the polar and azimuthal angles of the spherical coordinate frame whose center coincides with that of the particle and the $z$ axis with the propagation direction of the incident wave; $P_{\ell}^{(1)}(\cos \theta)$ stands for the associated Legendre polynomial, $P_{l}^{(1)^{\prime}}(\cos \theta)=d P_{l}^{(1)}(\cos \theta) /$ $d(\cos \theta)$, scattering amplitudes $a_{l}$ and $b_{l}$ may be written in the following form:

$$
a_{\ell}=\frac{F_{\ell}^{(a)}(q, \epsilon)}{F_{\ell}^{(a)}(q, \epsilon)+i G_{\ell}^{(a)}(q, \epsilon)},
$$

and similar for $b_{\ell}$ with replacement $F^{(a)}, G^{(a)} \rightarrow F^{(b)}, G^{(b)}$, where $\epsilon(\omega)$ is the relative (with respect to the environment) dielectric permittivity of the particle, $q=k R, k$ stands for the wave number of the incident wave in vacuum, and $R$ for the particle radius. As for $F^{(a, b)}, G^{(a, b)}$, they are expressed in terms of the Bessel $\left[J_{l+1 / 2}(\zeta)\right]$ and Neumann $\left[N_{l+1 / 2}(\zeta)\right]$ functions. The corresponding expressions are cumbersome and may be found in Ref. [6]. The partial scattering cross section $\left(\sigma_{\text {sca }}^{(\ell)}\right)$, is connected with $a_{\ell}, b_{\ell}$ :

$$
\sigma_{\text {sca }}^{(\ell)}=\frac{2 \pi}{k^{2}}(2 \ell+1)\left(\left|a_{\ell}\right|^{2}+\left|b_{\ell}\right|^{2}\right) .
$$

We remind the reader briefly of the results of the analysis of the Mie solution (for more details see, e.g., [4] and references therein). Optical resonances are defined by the condition $G_{\ell}^{(a, b)}\left(q, \epsilon_{\ell}\right)=0$. For a small particle $(q \ll 1)$ the condition $G_{\ell}^{(b)}\left(q, \epsilon_{\ell}\right)=0$, regarded as an equation for $\epsilon_{\ell}$, does not have any real solutions, while solutions of the equation $G_{l}^{(a)}\left(q, \epsilon_{\ell}\right)=0$ have the form $\epsilon_{\ell}=-(1+$ $\ell) / \ell+O\left(q^{2}\right)$. For nondissipating materials these solutions determine the resonant frequencies $\omega_{\ell}$ through the dependence $\epsilon(\omega)$. For weakly dissipating materials the roots of the equation $G_{\ell}^{(a)}\left(q, \omega_{\ell}\right)=0$ have small imaginary parts. In this case the resonant frequencies are equal to the real parts of the corresponding roots. Next, at $q \ll 1$ amplitudes $b_{\ell}$ are small relative to $a_{\ell}$ and may be dropped. Regarding $F^{(a)}$, in this limit $F_{\ell}^{(a)}=O\left(q^{2 \ell+1}\right)$ and does not vanish anywhere but at the trivial point $\epsilon=1$; as for $G^{(a)}$, away from the close vicinity of the resonance frequencies it is of the order of one. All together that results in sharp optical resonances for $\sigma_{\text {sca }}^{(\ell)}$, with a typical symmetric Lorenzian (Breit-Wigner) profile [13]. 
However, such a profile is not always the case for $I_{\|, \perp}^{(s)}$. Let us first discuss the nondissipative $\operatorname{limit}(\operatorname{Im} \epsilon=0)$. Considering excitations of nonresonant modes as background scattering and bearing in mind the narrowness of the resonance lines in this case [4] we obtain that the corresponding nonresonant amplitudes $a_{\ell} \approx a_{l 0}$, where $a_{l 0}=-i F_{\ell}^{(a)}(q, \epsilon) / G_{\ell}^{(a)}(q, \epsilon)=O\left(q^{2 l+1}\right), \quad$ are purely imaginary. Then, the dipole mode $(\ell=1)$ always plays the dominant role in the background scattering. Accordingly, in the vicinity of the dipole resonance the intensity of the dipole scattering always remains large relative to the contribution of the other modes. As a result interference of the resonant scattering with the background produces only small corrections to the resonant mode and the profiles $I_{\|, \perp}^{(s)}(\epsilon)$ remain Lorentzian.

For higher order resonances with $\ell>1$, which are also well pronounced in the nondissipative limit even for a small particle [4], the situation is different qualitatively. In these cases the resonance scattering arises from the corresponding background, whose amplitude $a_{\ell 0}$ is much smaller than that for the background dipole mode $\left(a_{10}\right)$. As $\epsilon$ approaches $\epsilon_{\ell}$ the resonant amplitude rapidly increases and becomes much greater than $a_{10}$, reaching a maximum at $\epsilon=\epsilon_{\ell}$ and decreasing back to $a_{\ell 0}$ upon further change of $\epsilon$. Thus, in the vicinity of $\epsilon_{\ell}$ there are two points (one to the left, the other to the right of $\epsilon_{\ell}$ ) where $\left|a_{\ell}\right|=\left|a_{10}\right|$. Bearing in mind that passage of the resonance adds $\pi$ to the phase of the resonant mode it gives rise to the conclusion that one of the equal-amplitude points should correspond to the constructive, while the other to destructive interference; i.e., an asymmetric Fano resonance profile should be observed.

To illustrate this reasoning let us consider the vicinity of the quadrupole resonance $(\ell=2)$. Taking $F_{1,2}^{(a)}, G_{1}^{(a)}$ at $\omega=\omega_{\ell}$, employing expansions of the functions in powers of $q$ [4], and additionally expanding $G_{2}^{(a)}$ in powers of $\delta \epsilon=\epsilon-\epsilon_{2}$ [we remind the reader that $G_{2}^{(a)}\left(q, \epsilon_{2}\right)=0$ ] one obtains the following approximate expressions for $I^{(s)}$ :

$$
\begin{gathered}
I_{\|}^{(s)} \propto\left|i q^{3} \cos \theta+\frac{q^{5} \cos 2 \theta}{2\left(q^{5}-12 i \delta \epsilon\right)}\right|^{2}, \\
I_{\perp}^{(s)} \propto\left|i q^{3}+\frac{q^{5} \cos \theta}{2\left(q^{5}-12 i \delta \epsilon\right)}\right|^{2} .
\end{gathered}
$$

There are two characteristic scales in Eqs. (6) and (7), describing (i) a sharp resonant line shape centered at $\delta \epsilon=$ 0 with the width $\Gamma=q^{5} / 6$ [14] and (ii) practically full suppression of the scattering which occurs at much greater scale: $\delta \epsilon_{\|}=-q^{2} \cos 2 \theta / 24 \cos \theta$ and $\delta \epsilon_{\perp}=$ $-q^{2} \cos \theta / 24$, but still inside the resonance region $\left(\left|\delta \epsilon_{\|, \perp}\right| \ll 1\right)$. Note also that there is a change of sign of $\delta \epsilon_{\|, \perp}$ with variation in $\theta$ shifting the annihilation point from one side of the resonance peak to another through vanishing of the corresponding $I^{(s)}$.
Finite dissipation and/or increase in $q$ broaden the resonances. It results in the overlap of resonance lines of different orders, which eventually may produce very complex profiles. Nevertheless the phenomenon remains qualitatively the same as long as the dissipation is weak. Profiles of forward and backward scattering, calculated for a potassium nanoparticle immersed in a $\mathrm{KCl}$ crystal, are presented in Fig. 1 as an example. The calculations are performed for a realistic dependence $\epsilon(\omega)$ fitting actual experimental data. Note, that in accordance with Eqs. (6) and (7) points of the destructive interference for the forward $(\theta=0)$ and backward $(\theta=\pi)$ scattering lie on different sides of the corresponding resonant peaks [15].

One-dimensional model. - Next we study the resonant wave scattering in systems with reduced spatial dimensionality. Numerous examples of such systems could be found, e.g., in book [16]. Fano resonances exhibited by these systems have exactly the same nature as those discussed above. To verify it we consider a localized point defect (modeling a scattering particle) in a simple 1D discrete chain (modeling the environment) with the interaction between nearest and next-to-nearest neighbor sites. The nonlocal coupling is a fundamental feature of the modelit provides a scattered wave with the possibility of bypassing the defect which simulates diffraction.

The model is described by the following set of equations (see also Fig. 2), where $\phi$ stands for the electric field of light and integer $n$ labels the spatial sites:

$$
i \frac{d \phi_{n}}{d t}+\sum_{j=-2}^{2} C_{n+j}^{n} \phi_{n+j}=0 ; \quad C_{n}^{n}=\omega_{0} \delta_{n 0} \phi_{n} .
$$

All sites of the chain but a defect situated at $n=0$ are identical and the coupling is mirror symmetric $\left(C_{n}^{m}=C_{m}^{n}\right)$. Accordingly, $C_{n \pm 1}^{n}=1, C_{n \pm 2}^{n}=\gamma$ for $|n|>2$. As for the defect, $C_{ \pm 1}^{0}=\mu, C_{ \pm 2}^{0}=\mu \gamma$, where $\gamma$ and $\mu$ are real positive constants; $\omega_{0}$ stands for the shift of the defect intrinsic frequency with respect to that for the other sites of the chain. The latter is set to zero $\left(C_{n}^{n}=0\right.$ at $\left.n \neq 0\right)$.

The solvability condition for linear traveling wave solutions $\left[\phi \sim \exp \left(i \omega_{\kappa} t \pm i \kappa n\right)\right]$ in the defect-free region of Eq. (8) results in the following dispersion relation:

$$
\omega_{\kappa}=2(\cos \kappa+\gamma \cos 2 \kappa) \text {, }
$$

where $\kappa$ is a (quasi)wave number. To keep the one-to-one

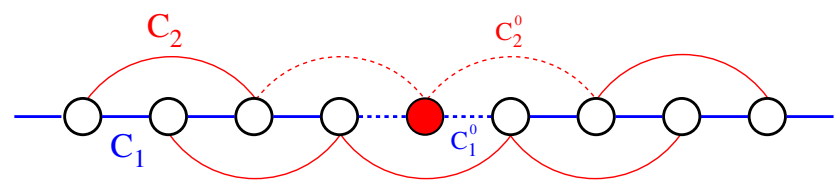

FIG. 2 (color online). Schematic view of a discrete 1D chain of sites with a defect described by Eq. (8). Solid and dashed lines in the vicinity of the defect correspond to the background and resonant scattering, respectively; see the text for more details. 
correspondence between $\omega_{\kappa}$ and $\kappa$ from the nontrivial range $0 \leq \kappa \leq \pi$ we impose the restriction $\gamma \leq 1 / 4$.

The defect-free region allows for another type of solutions whose permitted frequencies cover the entire band of those for Eq. (9): $-2(1-\gamma) \leq \omega_{\kappa} \leq 2(1+\gamma)$. These solutions are obtained by assigning in Eq. (9) $\kappa=\pi \pm$ $i p ; p \geq 0$. They correspond to exponentially decaying in space localized states.

To study the wave scattering by a defect in our toy model we consider an incident wave with amplitude being equal to one advancing from the left. Then, the corresponding boundary conditions read

$$
\phi_{n}=e^{i \omega_{\kappa} t} \begin{cases}e^{-i \kappa n}+\rho_{1} e^{i \kappa n}+\rho_{2} e^{p n}, & n<-2 \\ \tau_{1} e^{-i \kappa n}+\tau_{2} e^{-p n}, & n>2,\end{cases}
$$

where $\tau_{1}$ and $\rho_{1}, \tau_{2}$ and $\rho_{2}$ are the complex transmission and reflection amplitudes of the propagating and evanescent waves from both sides of the defect, respectively. The transmission coefficient $T$ is related to $\tau_{1}$ by the expression $T=\left|\tau_{1}\right|^{2}$

The linear problem (8)-(10) is exactly solvable, but the solution is extremely cumbersome. It is remarkable, however, that the expression for $\tau_{1}$ has a structure identical to that of Eq. (4), namely

$$
\tau_{1}=-\frac{F^{\left(\tau_{1}\right)}}{F^{\left(\tau_{1}\right)}+i G^{\left(\tau_{1}\right)}} ; \quad \operatorname{Im} F^{\left(\tau_{1}\right)}=\operatorname{Im} G^{\left(\tau_{1}\right)}=0 .
$$

Equation (11) is simplified drastically at small $\gamma, \mu$, and $\omega_{0}$ under the additional restriction of weak coupling between the defect and the chain: $\mu^{2} \ll\left|\omega_{0} \gamma\right|$. In this case

$$
\tau_{1} \simeq-\frac{\mu^{2}+\gamma \delta \omega}{\mu^{2}+\gamma \delta \omega-i \frac{\delta \omega}{2}} ; \quad \delta \omega \equiv \omega_{\kappa}-\omega_{0} .
$$

The corresponding $T(\delta \omega)$ has two scales: $T(\delta \omega) \rightarrow 1$, exhibiting a sharp spike with the linewidth $\Gamma \simeq 4 \mu^{2}$ centered at $\delta \omega=0$ (i.e., at $\omega_{\kappa}=\omega_{0}$ ) while it vanishes at much larger scale, at $\delta \omega=-\mu^{2} / \gamma$; cf. Eqs. (6) and (7). Note transformation of $T(\delta \omega)$ into a Lorenzian profile with reduction of the coupling to local at $\gamma \rightarrow 0$.

To be certain that the obtained properties of $T(\delta \omega)$ are related to the resonant excitation of the defect, we calculate $\left|\phi_{0}(\delta \omega)\right|^{2}$. At the small $\gamma, \mu$, and $\omega_{0}$ it has a typical Lorenzian profile (with the same linewidth $\Gamma \simeq 4 \mu^{2}$ ) overlapping the spike of $T(\delta \omega)$ : $\max \left|\phi_{0}(\delta \omega)\right|^{2} \simeq 1 / \mu^{2}$ is achieved at $\delta \omega \simeq-4 \gamma \mu^{2}\left(4 \gamma \mu^{2} \ll \Gamma\right)$.

More extensive analysis of Eqs. (8) -(10) restricted only by the constraint $\gamma \leq 1 / 4$ does not add any qualitative difference to the results discussed above, except for the fact that the locations of resonant transmission and reflection may be well separated in the frequency space.

Note, though the next-to-nearest site coupling is crucial to the model, its generalization to more extended interactions (provided the coupling constants become smaller with increase in distance between the interacting sites) gives rise just to small quantitative corrections to the model characteristics.
Conclusions. - A deep connection between the light scattering by a single finite-size obstacle and Fano resonances in quantum physics has been revealed and illustrated by the analysis of the exact Mie solution and a 1D toy model. The concept developed in the present Letter may be generalized to more complicated problems, e.g., the scattering by a system of particles including coherent backscattering and weak localization phenomena, scattering in a waveguide, etc. In each particular system, the specific resonant states are different but all of them act in the same way being described by similar physics.

We are grateful to Boris Rubinstein for help with the application of software MATHEMATICA to the analysis of Eqs. (8)-(10) and to Konstantin Kikoin for discussions of the results. This work has been partly supported by the Australian Research Council.

*tribelsky@mirea.ru

[1] M. Born and E. Wolf, Principles of Optics (Cambridge University Press, Cambridge, U.K., 1999), 7th ed.

[2] C.F. Bohren and D. R. Huffman, Absorption and Scattering of Light by Small Particles (Wiley, New York, 1998).

[3] W. L. Barnes, A. Dereux, and T.W. Ebbesen, Nature (London) 424, 824 (2003); S. A. Maier et al., Nat. Mater. 2, 229 (2003).

[4] M. I. Tribelsky and B. S. Luk'yanchuk, Phys. Rev. Lett. 97, 263902 (2006).

[5] M. Bashevoy, V. Fedotov, and N. Zheludev, Opt. Express 13, 8372 (2005).

[6] Z. B. Wang, B.S. Luk'yanchuk, M. H. Hong, Y. Lin, and T. C. Chong, Phys. Rev. B 70, 035418 (2004).

[7] U. Fano, Phys. Rev. 124, 1866 (1961).

[8] L. A. Falkovsky and S. Klama, Physica (Amsterdam) 264C, 1 (1996).

[9] S. Fan and J. D. Joannopoulos, Phys. Rev. B 65, 235112 (2002); G. Gantzounis and N. Stefanou, Phys. Rev. B 72, 075107 (2005).

[10] B. S. Luk'yanchuk, M. I. Tribelsky, Z. B. Wang, Y. Zhou, M. H. Hong, L. P. Shi, and T. C. Chong, Appl. Phys. A 89, 259 (2007).

[11] S. Flach, V. Fleurov, A. V. Gorbach, and A.E. Miroshnichenko, Phys. Rev. Lett. 95, 023901 (2005).

[12] S.F. Mingaleev, A.E. Miroshnichenko, Yu.S. Kivshar, and K. Busch, Phys. Rev. E 74, 046603 (2006).

[13] We should stress this is true only for small particles. If a particle is not small the line $\sigma_{\text {sca }}^{(\ell)}(\omega)$ may also have a typical asymmetric Fano resonance shape.

[14] The obtained expression for $\Gamma$ coincides with that given by Eq. (8) in Ref. [4] [owing to a misprint Eq. (8) in Ref. [4] misses the factor 2 in the numerator].

[15] The obtained small shift of the resonance peak with the variation in $\theta$ from 0 to $\pi$ is caused by the mentioned effects of finite $q$ and finite dissipation.

[16] M. J. Ablowitz, B. Prinari, and A. D. Trubatch, Discrete and Continuous Nonlinear Schrödinger Systems (Cambridge University Press, Cambridge, U.K., 2004).

[17] M. I. Tribel'skiǔ, Sov. Phys. JETP 59, 534 (1984). 